\title{
Oral Rehabilitation of a Severely Resorbed Edentulous Maxilla with Screwed-retained Hybrid Denture Using Cresco System: A Case Report
}

\author{
Ilser Turkyilmaza \\ Neal S. Patel ${ }^{b}$ \\ Edwin A. McGlumphyc
}

\begin{abstract}
Due to the angulation of maxillary bone as the maxillary bone resorption occurs from a buccal to palatal direction, bucco-lingually tilted implant placement is more likely especially in the posterior maxilla. The aim of this paper was to present a case restored by using a maxillary hybrid denture using Cresco method. A 59-year-old woman with an edentulous maxilla and five missing mandibular teeth was referred for implant treatment. Six months after sinus lift operation, four mandibular implants and six maxillary implants were placed. Due to the bucco-lingual tilt of two implants, the Cresco method was chosen to fabricate the metal framework for the maxillary hybrid denture that was delivered four months after implant placement. The main advantages of the Cresco method are a perfectly passive framework fit and corrected screw access holes. All ten implants placed were considered successful at 1-year recall, and no screw access holes were seen in the buccal part of the maxillary acrylic teeth. It was concluded that such screw-retained maxillary hybrid denture using the Cresco method met the esthetic expectations of a highly demanding patient. (Eur J Dent 2008;2:220-223)
\end{abstract}

Key words: Tilted implant; Hybrid; Maxilla; Early loading.

10 assistant Professor, Department of Prosthodontics, Dental School at San Antonio, University of Texas Health Science Center, San Antonio, Texas.

b Previous Implant Prosthodontic Fellow, Department of Restorative and Prosthetic Dentistry, College of Dentistry, The Ohio State University, Columbus, Ohio. c Associate Professor, Department of Restorative and Prosthetic Dentistry, College of Dentistry, The Ohio State University, Columbus, Ohio.

- Corresponding author: Dr. Ilser Turkyilmaz Department of Prosthodontics, Dental School, University of Texas Health Science Center at San Antonio, 7703 Floyd Curl Drive, MSC 7912, San Antonio, Texas 78229-3900

Phone: +1 2105676420 Fax: +1 2105676376

E-mail: turkyilmazQuthscsa.edu

\section{INTRODUCTION}

The presence of alveolar bone with sufficient volume and/or density is considered a prerequisite for implant placement, integration and load bearing, and subsequent good outcomes. ${ }^{1}$ However, bone resorption following tooth extraction or due to pneumatization of the maxillary sinus may cause inadequate bone in the horizontal and/or vertical dimension for dental implant placement. The augmentation of the maxillary sinus floor achieved by bone grafts placed inside the sinus cavity in order to create space for and accelerate bone formation 
is the most widely used method to re-establish adequate bone volume in the posterior maxilla. ${ }^{2,3}$

Fromananatomicstandpoint, the rehabilitation of edentulous maxilla is often complicated by poor bone quality and bone resorption from a buccal to palatal direction compared to the mandible. Therefore, tilted implant placement is sometimes required to create a Class I posterior occlusion. ${ }^{4}$ The use of tilted implants in the residual alveolar bone may has some clinical advantages: a) This method allows the placement of longer implants, which increase the implant-to-bone contact area and primary stability, b) Tilting the implant creates a wider distance between anterior and posterior implants, which result in better load distribution, c) This method reduces or eliminates the need for a cantilever in the prosthesis.

The Cresco method (Cresco-Ti Precision Technique; Cresco-Ti Co, Krinstianstad, Sweden) is a new way of fabricating a metal framework for fixed implant-supported prostheses to eliminate the unavoidable distortions created while casting the framework. This new method implies a horizontal sectioning of the cast framework. The coronal part of the framework is thereafter attached by a laser welding technique to new premachined cylinders mounted on a master cast. The coronal surfaces of the cylinders are cut in the same horizontal plane as the lower surface of the framework. ${ }^{5}$

Moreover, the immediate/early loading of implants placed in the fully edentulous maxilla to support fixed prosthesis is one of the most remarkable achievements in implant dentistry and clinical studies have recently showed encouraging results with early loading protocols of implants in the maxilla., ${ }^{6,7}$

The aim of this paper was to describe a case restored by using a maxillary hybrid denture supported by both upright and tilted implants due to the angulation of maxillary bone as the maxillary bone resorption occurred from a buccal to palatal direction.

\section{CASE REPORT}

A 59-year-old woman with an edentulous maxilla and five missing mandibular teeth was referred for implant treatment. Intraoral examination and the panoramic radiograph of the patient showed bilateral sinus cavity enlargement, considerable maxillary bone resorption both horizontally and vertically resulting in a poor esthetic appearance (Figure 1).

Both maxillary left and right sinus lifting operations were performed (Bio-Oss $®$, Geistlich Pharma AG, Wolhusen, Switzerland). An incision was made on the alveolar crest in the edentulous area. The bone window was created by removing all cortical bone up to the sinus membrane. After the membrane was exposed, the graft was placed into the sinus cavity. An absorbable collagen membrane (Bio-Gides, Geistlich Pharma AG, Wolhusen, Switzerland) was then placed on the lateral wall of the sinus to avoid migration of the graft and its invasion by soft tissues. After the sinus lift operation, the maxillary temporary complete denture was delivered to the patient following soft relining.

Four mandibular implants (two $4.5 \times 11 \mathrm{~mm}$, one $4.5 \times 13 \mathrm{~mm}$, one $4.0 \times 11 \mathrm{~mm}$; Astra OsseoSpeed, Mölndal, Sweden) and six maxillary implants (2 buccolingually tilted and 4 straight) (five 4.0x13 $\mathrm{mm}$, one $4.0 \times 11 \mathrm{~mm}$ ) were placed six months after the sinus lift operation. The gingival formers were screwed on top of the implants four months after implant placement. The final impressions were made, and master casts were poured. The master casts were mounted on the articulator using a face-bow. A previously fabricated silicone teeth key was seated on the maxillary master cast. Because of the bucco-lingual tilt of the two implants, the Cresco method (Cresco-Ti Precision Technique; Cresco-Ti Co, Krinstianstad, Sweden) was chosen to fabricate the metal framework for the maxillary hybrid denture (Figure 2). Straight or individually angulated (by heat) acrylic tubes were mounted on the implant analogues in the master cast by the help of process screws, followed by a conventional wax-up. The acrylic tube was bent in a palatal direction for the two tilted implant positions, so the retention screw entrance did not penetrate the facial part of the teeth of maxillary hybrid denture. The main advantages of the Cresco method are a perfect passive fit and corrected screw access holes which allow the most advantageous placement of the implant. The maxillary hybrid denture was screwed onto the unique abutments used only for the Cresco system and mandibular implant-supported restorations (Ceramco, Dentsply, Burlinton, NJ) 
were cemented the same day (Figures 3 and 5). Unilaterally balanced occlusion was used. All ten implants placed were successful one year after implant placement, which was confirmed by a panoramic radiograph (Figure 5). No screw access holes were seen in the buccal part of the maxillary acrylic teeth and no fitting problem was noted during metal framework try-in.

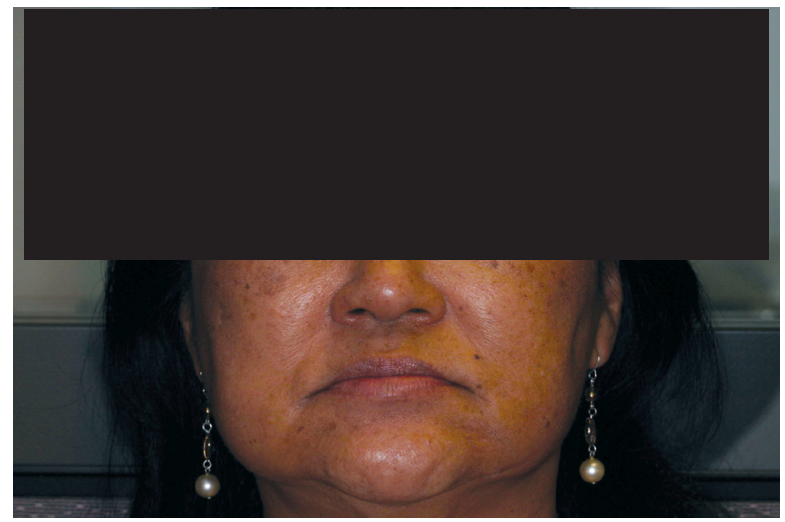

Figure 1. Frontal extra-oral view of the patient prior to the implant placement.
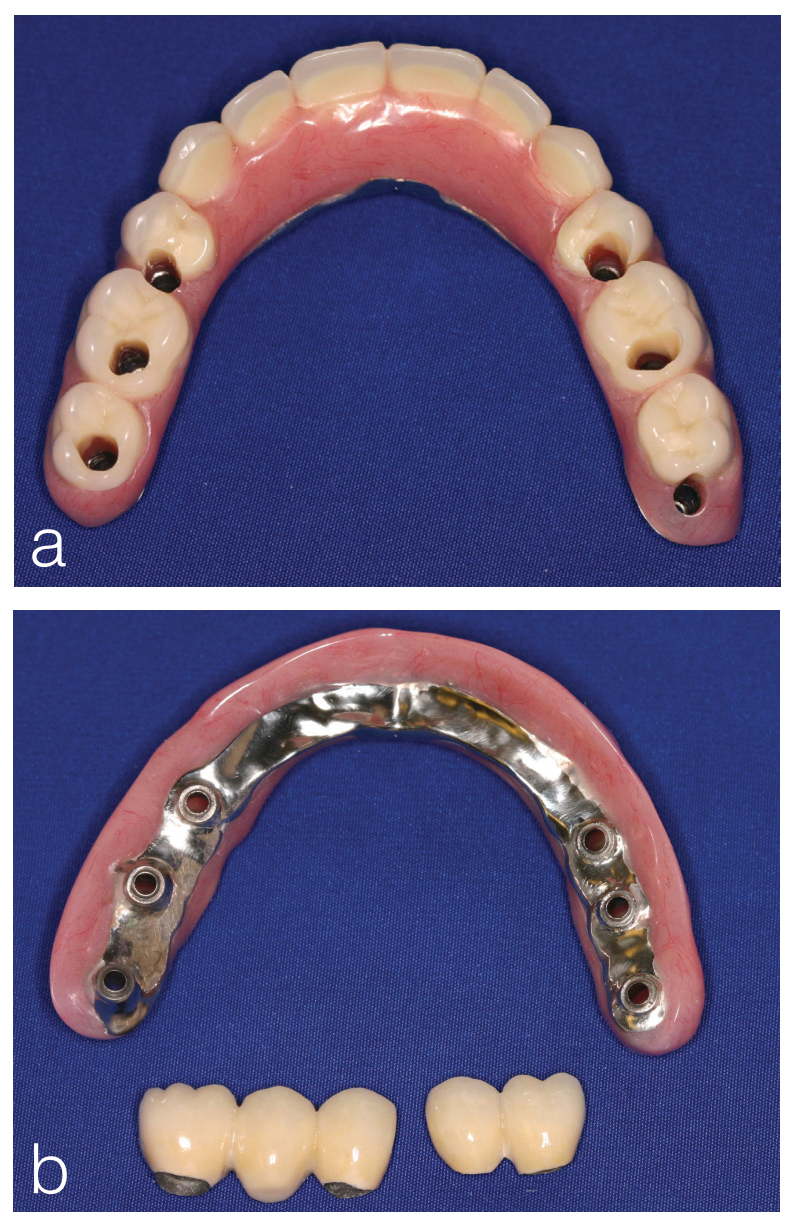

Figure 2. Occlusal (a) and gingival (b) views of the maxillary hybrid denture with corrected screw holes fabricated using the Cresco method.

\section{DISCUSSION}

Because dental implants are integrated into bone, passive fit between implant and superstructure decreases the risks of biomechanical stress development that may negatively influence implant survival. ${ }^{8}$ The main advantages of the Cresco method are a perfect passive fit and corrected screwaccess holes. Thus, Cresco provides maximum flexibility in terms of being able to compensate for angled implant placement when bone quality and quantity are poor. This eliminates the risk of buccal access

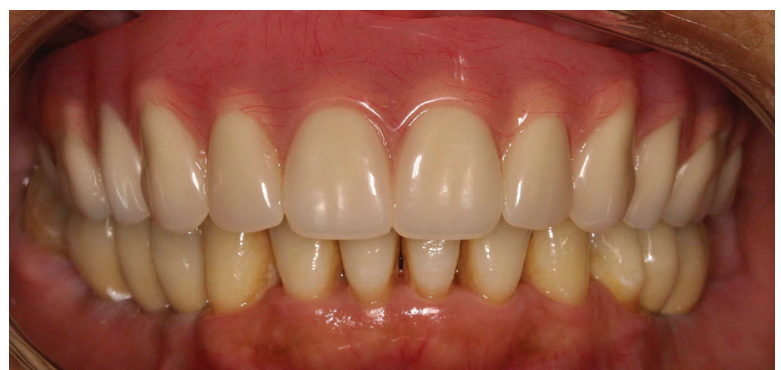

Figure 3. Intra-oral view of the patient after maxillary hybrid denture and mandibular implant-supported restorations were seated.

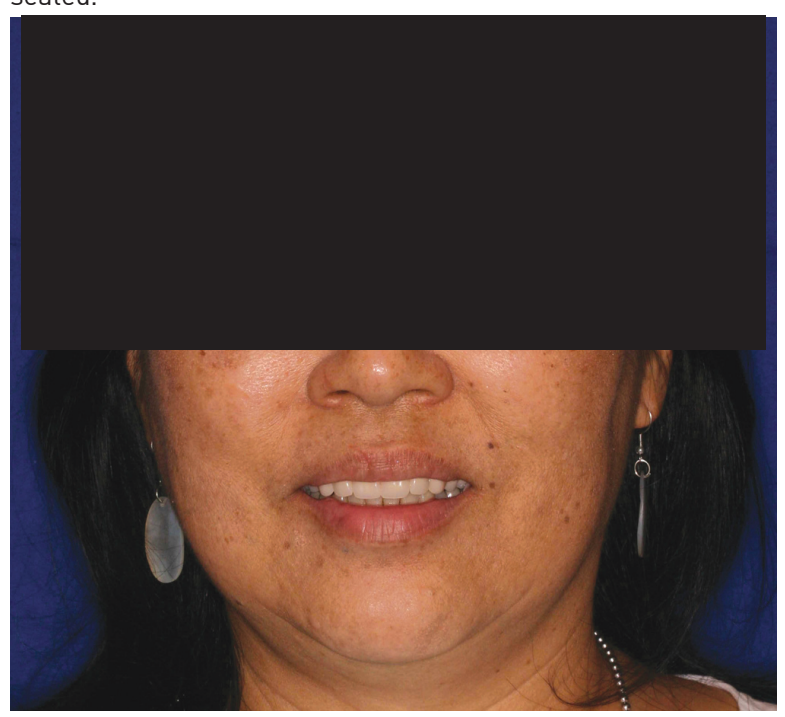

Figure 4. Frontal extra-oral view of the patient after the restorations were placed.

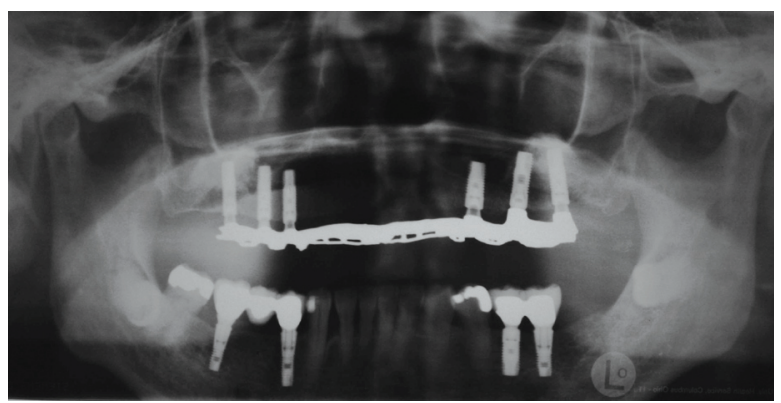

Figure 5. Panaromic radiograph after one year. 
holes compromising esthetic result.

The report by Hellden et $a^{1}{ }^{8}$ described the Cresco bridge including innovative and simplified clinical and laboratory procedures for the fabrication of abutment-free, cast titanium superstructures with passive fit to implants. They concluded that the Cresco superstructure provided the precision fit between the implants and superstructures.

Another study by Hellden et al, ${ }^{9}$ investigated the clinical and radiographic outcome of a prospective 5-year longitudinal multicenter test of a simplified implantology concept comprising an abutment-free implant system (Cresco) and a new method for fabrication of passively fitting superstructures (Cresco Ti Precision method). 60 partially or completely edentulous patients with 215 implants were restored by fixed implantsupported superstructures fabricated using the Cresco Ti Precision method. The radiographic measurements were recorded from radiographs exposed perpendicular to the implants. The initial implant failure rate (during the healing phasel was $2 \%$, and the survival rate after loading was $98 \%$. The mean peri-implant bone loss was $0.29 \mathrm{~mm}$. Of the measured sites, $71 \%$ showed a crestal bone loss of less than 0.5 $\mathrm{mm}$. Very few mechanical complications were observed. This was attributed to the passively fitting superstructures. They concluded that the abutment-free Cresco implantology concept is a reliable alternative method for implant-supported fixed prosthetic rehabilitation of edentulous and partially edentulous jaws.

\section{CONCLUSIONS}

It was concluded that such screw-retained maxillary hybrid denture using Cresco method may be a viable option to restore edentulous maxilla, and no framework fitting problems were noted.

\section{REFERENCES}

1. Chen TW, Chang HS, Leung KW, Lai YL, Kao SY. Implant placement immediately after the lateral approach of the trap door window procedure to create a maxillary sinus lift without bone grafting: a 2-year retrospective evaluation of 47 implants in 33 patients. J Oral Maxillofac Surg 2007;65:2324-2328.
2. Cheng AC, Tee-Khin N, Siew-Luen C, Lee H, Wee AG. The management of a severely resorbed edentulous maxilla using a bone graft and a CAD/CAM-guided immediately loaded definitive implant prosthesis: a clinical report. $J$ Prosthet Dent 2008;99:85-90.

3. Becktor JP, Hallström H, Isaksson S, Sennerby L. The use of particulate bone grafts from the mandible for maxillary sinus floor augmentation before placement of surface-modified implants: results from bone grafting to delivery of the final fixed prosthesis. J Oral Maxillofac Surg 2008;66:780-786.

4. Capelli M, Zuffetti F, Del Fabbro M, Testori T. Immediate rehabilitation of the completely edentulous jaw with fixed prostheses supported by either upright or tilted implants: a multicenter clinical study. Int J Oral Maxillofac Implants 2007;22:639-644.

5. Hjalmarsson L, Smedberg Jl. A 3-year retrospective study of Cresco frameworks: preload and complications. Clin Implant Dent Relat Res 2005;7:189-199.

6. Turkyilmaz I, Avci M, Kuran S, Ozbek EN. A 4-year prospective clinical and radiological study of maxillary dental implants supporting single-tooth crowns using early and delayed loading protocols. Clin Implant Dent Relat Res 2007;9:222-227.

7. Testori T, Del Fabbro M, Capelli M, Zuffetti F, Francetti L, Weinstein RL. Immediate occlusal loading and tilted implants for the rehabilitation of the atrophic edentulous maxilla: 1-year interim results of a multicenter prospective study. Clin Oral Implants Res 2008;19:227-232.

8. Helldén LB, Ericson G, Olsson CO. The Cresco Bridge and implant concept: presentation of a technology for fabrication of abutment-free, passively fitting superstructures. Int $J$ Periodontics Restorative Dent 2005;25:89-94.

9. Helldén L, Ericson G, Elliot A, Fornell J, Holmgren K, Nilner K, Olsson CO. A prospective 5-year multicenter study of the Cresco implantology concept. Int J Prosthodont 2003;16:554-562. 